Books, videos, CD-ROMs, DVDs and any other relevant items submitted for a review in the $B D J$ should be addressed to: Kate Maynard, Assistant Editor, British Dental Journal, NPG, 4-6 Crinan Street, London N1 9XW

\section{Integrated dental treatment planning: A case-based approach}

\author{
E.J. Kay, A.C. Shearer, A.M. Bridgman, G.M. \\ Humphris \\ UK: Oxford University Press \\ price $€ 24.95$, pp 159
}

ISBN 0198528892

This remarkable book - which has the shortest contents page I have ever seen - is not about dentistry but about people.

In contrast to traditional textbooks this book plots the dental history of six people over a period of some years. Each case is very different, but all incorporate material that is seen in everyday clinical practice.

The book challenges you to consider clinical, psychological, social, legal and ethical issues while reading each of the six case studies. The authors want us to think about these issues in the context of planning and providing dental treatment and they have certainly succeeded in my opinion. The book is interactive, well-structured and incredibly easy to read. Readers will particularly like how each story is drip fed to them, giving them opportunity to reflect and consider before more of the story is revealed.

Conceptually, treatment planning is difficult to grasp and all too frequently practitioners focus on dental factors to the exclusion of other equally important factors, for example the psychological, social, or legal aspects as they relate to the patient's overall management. This book takes you beyond the normal limits of traditional dental textbooks and will no doubt cause every reader to stop, think and either agree or disagree with the thoughts of the authors, or better still stimulate further debate and discussion of the various issues raised.

This book particularly encourages a holistic and integrated approach and asks practitioners to 'think outside the box'. The style of this book and the authors' approach is refreshing and will appeal to a wide readership. The book is suited to final year dental students and practitioners alike and is to be strongly recommended.

\section{Endodontics}

C.J.R. Stock, R.T. Walker, K. Gulabivala
UK: Mosby
price $€ 120$, pp 324
ISBN 0723432031

This is the third edition of the immensely popular text that started life as A colour atlas and text of endodontics. It is written by three well known clinically-orientated endodontists and this background is reflected in the pragmatic and detailed accounts of new endodontic techniques and theories. The text is lavishly illustrated with over 1,400 illustrations, many in colour.

The book aims to lead undergraduates and GDPs logically through the subject of endodontics. It achieves this starting with chapters on the rationale for treatment, preoperative considerations, preparation and obturation of the root canal then finishing with more complicated scenarios such as perio-endo lesions, cases requiring surgery and retreatment. Finally, details of restoring the endodontically treated tooth and endodontic treatment of primary teeth are covered as well as medico-legal issues.

The logical progression of the book makes it easy to read and the illustrations more often than not add to understanding of various concepts. The authors have obviously consulted widely on the content of the book and this has paid dividends. In particular, I liked the authors' concentration on infection control and the biofilm nature of endodontic infection; and their coverage of new concepts in endodontics including ultrasonics, greater taper nickel titanium files and thermoplastic obturation techniques.

In summary this book is a good overview of contemporary endodontics and would be useful to undergraduate students as well as GDPs, particularly if considering updating endodontic knowledge. Good overviews are provided of perio-endo considerations, root resorption and restoration of the roottreated tooth that would also be useful as a summary to the postgraduate student.

\section{Oral pathology}

\author{
J.V. Soames, J.C. Southam \\ UK: Oxford University Press \\ price $€ 39.95$, pp 278 \\ ISBN 0198527942
}

A good understanding of pathology is required prior to treating a condition. Once the mechanism and pathological process of a disease is understood, the likely outcome of any treatment provided to the patient may be determined. Oral pathology has always been considered a difficult subject for undergraduate dental students. This text, now in its twentieth year, has widely been cited as 'essential reading' in many UK and international dental curricula.

The contents follow a logical progression through 17 chapters, covering conditions affecting teeth, bone, oral mucosa and salivary glands. Each chapter outlines the essential details behind conditions such as odontogenic cysts and tumours, offering explanations on their proposed aetiology, pathogenesis and histological features. Information on recent advances in molecular biology and genetics as well as rare conditions are confined to additional information boxes so as not to overburden the reader with text.

As in previous editions the text is comprehensive and easy to follow. The layout is largely unchanged but with an increased number of useful key points to summarise conditions into quick glance notes: a recommended bonus for the busy practitioner. The use of good clinical, radiographic and histological colour photographs for the more 'common' conditions allow the reader to 'follow' the patient from presentation to the diagnostic stage.

This text is reasonably priced given its contents and extensive colour plates and tables. Oral pathology should retain its place as 'essential reading' in all dental curricula. Although this book is aimed at undergraduate dental students, I would not hesitate to recommend it as a useful book for general dental practitioners and postgraduate students aiming to further their knowledge in the field of oral pathology. 

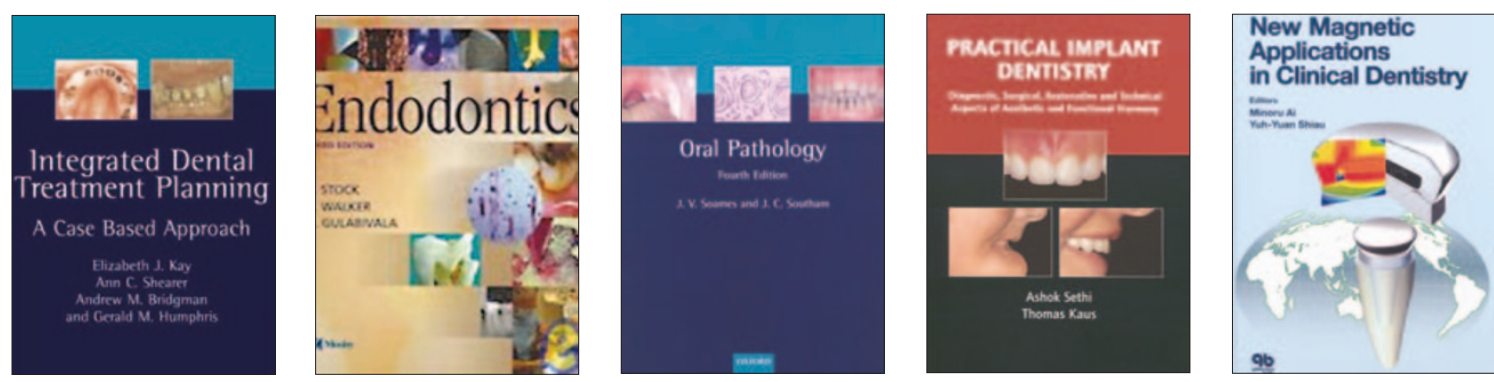

\section{Practical implant} dentistry - diagnostic, surgical, restorative and technical aspects of aesthetic and functional harmony

A. Sethi, T. Kaus

UK: Quintessence

price $€ 98$, pp 284

ISBN 1850970610

This beautifully illustrated hardback would be a welcome addition to any implantologist's library. The stated aims are to provide a 'Practical and pragmatic approach ... to present guidelines to assist the clinician during decision making...' These are admirably achieved.

The book is divided into three sections: Introduction and assessment; Implant placement and Augmentation. Each section contains a large number of photographic plates, clear diagrams and useful preoperative assessment checklists and flowcharts to facilitate decision-making. The book concentrates on the practicalities of implantology rather than discussing in depth the principles underpinning the techniques. The chapters are referenced throughout and the multiple headings make the text easy to follow. The implant system used is slightly unclear, but there are references to Ankylos.

Section one contains two very brief chapters on patient selection and medical history, but more than compensates for this with two comprehensive sections on additional diagnostic procedures (plain films, CT, MRI, ridge mapping and study casts) and anatomic variations.

Section two takes the reader step by step through all the clinical and technical stages of implantology from initial assessment, tooth extraction and impressions to implant placement, try in of the prosthetic framework and restoration completion. The section is subdivided into immediate placement, delayed placement and prosthetic protocols. Of particular interest is the advice on technique options for differing clinical scenarios. There are several diagrams outlining variations in incision design and suturing technique - two very important surgical considerations that are all too often overlooked. Each chapter finishes with case studies to further illustrate the techniques discussed.

Section three covers the topic of soft and hard tissue augmentation. Bone expansion, localised and iliac crest bone grafts are discussed in glorious technicolour detail. Neural repositioning and sinus lifts with their associated complications are described with references to instrumentation, radiographs, clinical photographs and CT scans.

In summary, this is an excellent book on practical implantology for those with a basic understanding of the subject. It will benefit a wide spectrum of clinicians from junior surgeons to experienced implantologists. However, it does not, nor does it claim to, contain information on the various implant systems available on the market or the fundamentals of osseointegration.

G. Ainsworth

\section{New magnetic applications in clinical dentistry}

M. Ai, Y-Y.Shiau

UK: Quintessence

price $€ 50$, pp 184

ISBN 4874178286

This is a unique, very interesting book on a subject that has received little attention in recent years. The authors are 15 Japanese academics, a director of the Aichi Steel Corporation of Japan and Harold Preiskel from the UK. The book is an updated version of a text originally published in Japanese in 1994. As a consequence, perhaps, of the translation, some of the spelling and English is somewhat inconsistent. The book is nevertheless very well presented and beautifully illustrated. It is divided into three parts: Fundamentals of magnet and magnetic attachment; Clinical application of magnetic attachment; and Clinical cases.

The use of magnets in prosthetic dentistry is not new but after early reports in the 1980s rather fell from favour as a consequence of problems experienced with earlier types of magnet. These problems related to their size, low retentive force and corrosion. It is claimed by one of the editors of the book that these problems were completely solved in Japan in 1990 and this book provides a comprehensive exposition of new developments since then. The breakthrough appears to have come with the replacement of samarium-cobalt magnets with neodymium-iron magnets which are more powerful and can be made much smaller. The problem of corrosion has been overcome by micro-laser welding corrosion-resistant stainless steel casings for the magnets. Root keepers can now be fixed directly to the root surface of abutments either by the use of adhesive restorative materials or with systems where the keeper forms part of a cast or prefabricated restoration.

Every aspect of health and safety, biological effects of magnets on the body and possible influences of magnetic attachments on medical devices is explored. The advantages and disadvantages are fully covered as is the concept of designing dentures with magnetic attachments. It is disappointing that the only follow-up data on the use of such devices is based on a relatively small number of patients (77) treated by different operators at Osaka University Dental Hospital. Over an eight year period the most commonly encountered problems were denture fractures, dislodgement of the magnetic assembly from the denture base and dislodgement of keepers from copings.

In the final section of the book, 25 cases of conventional removable partial dentures and two cases of implant retained dentures using magnetic attachments are presented. There is also a comprehensive listing of all dental magnet attachments that are sold in Europe and the USA.

This book is an extremely useful source of reference for anyone contemplating the use of magnetic retention either for conventional or implant retained prostheses.

M. Barsby 